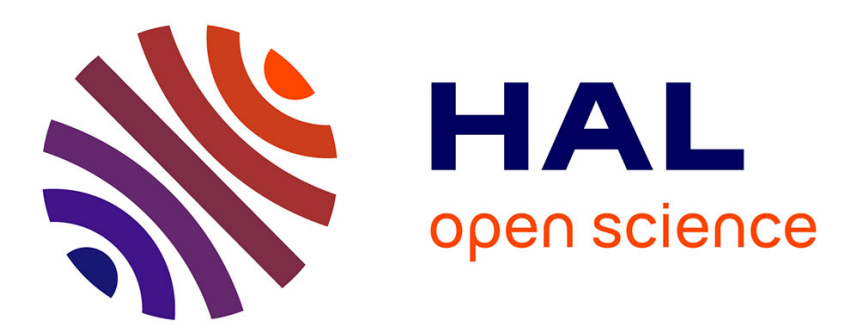

\title{
Correlation between structural and optical properties of WO3 thin films sputter deposited by glancing angle deposition
}

Cédric Charles, Nicolas Martin, Michel Devel, Julien Ollitrault, Alain Billard

\section{- To cite this version:}

Cédric Charles, Nicolas Martin, Michel Devel, Julien Ollitrault, Alain Billard. Correlation between structural and optical properties of WO3 thin films sputter deposited by glancing angle deposition. Thin Solid Films, 2013, 534, pp.275 - 281. 10.1016/j.tsf.2013.03.004 hal-00875712

\author{
HAL Id: hal-00875712 \\ https://hal.science/hal-00875712
}

Submitted on 22 Oct 2013

HAL is a multi-disciplinary open access archive for the deposit and dissemination of scientific research documents, whether they are published or not. The documents may come from teaching and research institutions in France or abroad, or from public or private research centers.
L'archive ouverte pluridisciplinaire HAL, est destinée au dépôt et à la diffusion de documents scientifiques de niveau recherche, publiés ou non, émanant des établissements d'enseignement et de recherche français ou étrangers, des laboratoires publics ou privés. 
1 Correlation between structural and optical properties of $\mathrm{WO}_{3}$ thin films sputter

Cédric CHARLES ${ }^{\text {a }}$, Nicolas MARTIN ${ }^{\text {a, }}$, Michel DEVEL ${ }^{\text {a }}$, Julien OLLITRAULT ${ }^{\text {a }}$,

Alain BILLARD ${ }^{b}$

6

${ }^{a}$ Institut FEMTO-ST, UMR 6174 CNRS, Université de Franche-Comté, ENSMM, UTBM

32, Avenue de l'observatoire, 25044 BESANCON Cedex, France

9

${ }^{\mathrm{b}}$ LERMPS, UTBM, Site de Montbéliard, 90010 BELFORT Cedex, France

${ }^{1}$ Author to whom correspondence should be addressed: Tel.: +33 (0)3 818539 69; Fax: +33 (0)3 81853998 ; Email: nicolas.martin@femto-st.fr 


\section{Abstract}

Tungsten oxide $\mathrm{WO}_{3}$ thin films are prepared by dc reactive sputtering. The GLancing Angle Deposition method (GLAD) is implemented to produce inclined columnar structures. The incident angle $\alpha$ between the particle flux and the normal to the substrate is systematically changed from 0 to $80^{\circ}$. For incident angles higher than $50^{\circ}$, a typical inclined columnar architecture is clearly produced with column angles $\beta$ well correlated with the incident angle $\alpha$ according to conventional relationships determined from geometrical models. For each film, the refractive index and extinction coefficient are calculated from optical transmittance spectra of the films measured in the visible region. The refractive index at $589 \mathrm{~nm}$ drops from $n_{589}=2.18$ down to 1.90 as $\alpha$ rises from 0 to $80^{\circ}$, whereas the extinction coefficient reaches $k_{589}=4.27 \times 10^{-3}$ for an incident angle $\alpha=80^{\circ}$, which indicates that the films produced at a grazing incident angle become more absorbent. Such changes of the optical behaviours are correlated with changes of the microstructure, especially a porous architecture, which is favoured for incident angles higher than $50^{\circ}$. Optical band gap $E_{g}$, Urbach energy $E_{u}$ and birefringence $\Delta n_{617}$, determined from optical transmittance measurements, are also influenced by the orientation of the columns and their trend are discussed taking into account the disorder produced by the inclined particle flux.

\section{Keywords}

$\mathrm{WO}_{3}$ films, GLAD, inclined columns, refractive index, porosity, optical band gap, Urbach energy, birefringence. 


\section{Introduction}

Transition metal oxides represent a very attracting class of materials because of the wide range of physical and chemical properties that they exhibit. Among these oxide compounds, tungsten oxide thin films have been extensively investigated due to their important applications as active layers for electrochromic window devices [1-4], sensors for toxic gases [5-8], optical coatings with high refractive index $[9,10]$ or transparent and low resistive oxide materials $[11,12]$. It is well known that many chemical and physical characteristics of metal oxide thin films are strongly connected to their chemical composition, especially the oxygen-to-metallic concentrations ratio, which can be tuned in order to get a metallic, semi-conducting or insulating behaviour according to the metalloid content in the film [13-16]. However, playing with the chemical composition is not the only approach to tune the properties of metal oxide thin films. The structure at the sub-micrometric scale can also influence the film performances for many applications [17]. So, the design and the growth control of nanostructures in thin layers appear as important issues, e.g. in order to control the optical properties by playing on structural features. To this aim, various strategies have been proposed for the structuration of thin films [18].

In the last decade, the interest of nanostructuration by evaporation and/or sputtering techniques was particularly boosted by the GLancing Angle Deposition (GLAD) method [19]. This method is based on the preparation of thin films on fixed or mobile substrate, with an oblique incidence of the incoming particle flux. Indeed, when the atomic vapour flow comes up at a non normal incident angle $\alpha$, the nucleation sites intercept the flow of particles. This creates a shadowing effect and there is a tilted grain growth of columnar shape leading to inclined columnar structures with an angle $\beta$ with respect to the normal of the substrate surface. Nature, crystallography, temperature and surface conditions of the substrate, energy and interactions of the condensed particles with the substrate, among other parameters, have a decisive role in the growth mode of the coating. As a result, the GLAD technique can control the structure of thin films at the micro- and nanoscales. The experimental setup has two degrees of freedom: a rotation axis at an angle $\alpha$, which allows to vary 
the incident angle of the particle flux, and a rotary axis at an angle $\phi$ (also called azimuth angle), which modifies in an indirect way, the position of the particle source. The produced architectures can be of type i) columnar and inclined; ii) chevron or zigzag by alternating periodically the incident angle of particles from $+\alpha$ to $-\alpha$ maintaining constant $\phi$ angle (azimuthal angle around the substrate) or with a $180^{\circ}$ rotation of $\phi$ keeping constant $\alpha$ angle; iii) spiral or helical thanks to a continuous rotation of $\phi$ at a constant incident angle $\alpha$. This latter type adds to the potential of the GLAD technique. Morever, changing wisely $\alpha$ and $\phi$ angles as well as speeds of rotation, more original structures can be obtained such as porous columnar structures with variable diameters [20] or helical columns with squared sections [21]. In the end, the GLAD technique exploits the effects of shadowing created by a tilted substrate relative to normal incidence and a change of the direction of the particle flux through a rotation of the same substrate during the deposition. The two combined can generate different forms of columns and varied architectures. For example, Robbie et al. [22, 23] or Van Popta et al. [24] have deposited by evaporation some structured films with columnar architectures showing sinusoidal, helical and more complex forms. This variety allows envisaging applications in many fields such as biomedical system [25], photonic devices [26], microsensors [27], etc. Moreover thin films deposited by GLAD have high porosity and anisotropic behaviours, which can be used as rugate filters [28], wavelength-selective polarizer [29], or antireflection coating [30].

The purpose of this article is to study the structural and optical properties of the sputter deposited tungsten oxide $\mathrm{WO}_{3}$ nanostructured thin films grown using various incident angles $\alpha$ of the particle flux from 0 to $80^{\circ}$. We systematically investigate how the structure and optical properties (refractive index, extinction and absorption coefficients, optical band gap, birefringence) of such oriented thin films can be tuned by changing the incident angle of the sputtered particles. The evolution of the porous structure connected to the columnar orientation is especially analyzed in order to discuss and understand some relationships between the architecture of the films and their resulting optical behaviours. 


\section{Experimental details}

$\mathrm{WO}_{3}$ films were sputter deposited by DC reactive magnetron sputtering using a home made system $[31,32]$. A tungsten target $(5 \mathrm{~cm}$ diameter with purity 99.9 at. \%) was powered at a constant current density $J=25.5$ A.m ${ }^{-2}$, with an argon partial pressure $P_{A r}=0.1 \mathrm{~Pa}$ and an oxygen partial pressure $P_{\mathrm{O} 2}=0.08 \mathrm{~Pa}$. Substrates (grounded and kept at room temperature) were glass plates and (100) silicon wafers. The distance between the target and the substrate was fixed at $60 \mathrm{~mm}$. The growth of the films was stopped at a thickness close to $1 \mu \mathrm{m}$ thanks to the calibration of the deposition rate. A systematic change of the incident angle from $\alpha=0$ to $80^{\circ}$ with a $10^{\circ}$ increment was performed to tune the inclined columnar structure. Films deposited on glass substrates were characterized thanks to optical transmittance spectra measured with a Lambda 900 Perkin Elmer spectrophotometer in the visible range from 1.55 to $3.10 \mathrm{eV}$ (i.e. wavelength in-between 800 to $400 \mathrm{~nm}$ ). Refractive index, extinction coefficient and absorption coefficient were determined from interference fringes obtained with experimental optical transmittance spectra using Swanepoel's method [32]. Films prepared on (100) silicon wafers were cross-sectioned and observed by field effect scanning electron microscopy (SEM) using a JEOL $6400 \mathrm{~F}$. $\mathrm{WO}_{3}$ structures were also characterized by X-ray diffraction (XRD). Measurements were carried out using a Bruker D8 focus diffractometer with a cobalt X-ray tube $\left(\mathrm{Co} \lambda_{K \alpha}=1.78897 \AA\right)$ in a $\theta / 2 \theta$ configuration.

\section{Results and discussion}

\subsection{Structural characterization}

Tungsten oxide thin films prepared with an incident angle $\alpha$ lower than $50^{\circ}$ do not exhibit a clear inclined columnar structure. A densely packed feature is rather observed with a smooth surface topography. However, a further increase of the incident angle ( $\alpha$ higher than $\left.50^{\circ}\right)$ leads to a rougher film/air interface and a more defined columnar growth. Observations by SEM of surfaces and crosssections of $\mathrm{WO}_{3}$ thin films sputter deposited with an incident angle $\alpha$ of 70 and $80^{\circ}$ are shown in 
figure 1. It is worth of noting that the top of the columns has a rather sharp appearance (Fig. 1a), which is even more emphasized for $\alpha=80^{\circ}$ (surface state becomes irregular and more voided as illustrated in figure 1c). Such increase of the surface roughness versus incident angle of the sputtered particles is in agreement with previous investigations focused on metal oxide coatings produced by GLAD $[33,34]$. It is mainly attributed to the shadowing effect at the atomic scale, which prevails over the surface diffusion of adatoms as the incident angle rises. The structural anisotropy (formation of growth islands connected to each other by chains perpendicular to the plane of incidence) previously claimed by Tait et al. [35], is slightly marked for sputtered tungsten oxide films. The top of the columns appears more or less connected to each other according to the $\mathrm{x}$ direction and perpendicular to the particle flux (Fig. 1a and 1c).

Inspection of the cross-sectional view ensures that the GLAD $\mathrm{WO}_{3}$ films are composed of slanted columns and inter-columnar voids (Fig. 1b and 1d). The columns are inclined towards the direction of the incoming vapour flux. The column angle $\beta$, defined as the angle between the substrate surface normal and the long axis of the slanted columns, is measured from the cross-section SEM images. For incident angle $\alpha$ lower than $50^{\circ}$, the column angle $\beta$ can not be accurately determined since no clear columnar growth has been produced but a densely packed structure. For higher angles of incidence $\left(\alpha>50^{\circ}\right)$, SEM images exhibit morphologies composed by columns and inter-columnar gaps. The columns become increasingly separated and can easily be distinguished at an incident angle $\alpha$ of $70^{\circ}$ and even more at $80^{\circ}$. The resulting column angles $\beta$ are 50 and $54^{\circ}$ for incident angles $\alpha$ of 70 and $80^{\circ}$, respectively. Such column angles deviate from the empirical tangent rule [36], which predicts 53 and $70^{\circ}$, respectively. This rule provides a first order approximation of the expected $\beta$ angles. Since the growth can be disturbed by many parameters (temperature, particle energy, pressure), the tangent rule fails to well describe experimental column angles, especially for grazing incident angles. This is indeed relevant for thin films deposited by the sputtering process, where column angles are often lower than those calculated with various ballistic rules [37, 38]. However, our produced $\mathrm{WO}_{3}$ column angles are in good agreement with relationships proposed by 
Tait et al. [39]. The sputtering pressure required to maintain the glow discharge restricts the mean free path of the sputtered particles and thus, reduces the shadowing effect. As a result, the theoretical column inclinations predicted by the simple tangent rule is systematically overestimated. Since tungsten oxide thin films have been deposited at room temperature (substrate temperature is lower than 0.3 times the melting point of $\mathrm{WO}_{3}$ compound), one could expect a poorly crystallized material. However, XRD analyses exhibit diffracted signals (Fig. 2). Peaks corresponding to the $\mathrm{WO}_{3}$ monoclinic structure are clearly identified for incident angles included between $\alpha=0$ and $80^{\circ}$. For normal incidence $\left(\alpha=0^{\circ}\right)$, as-deposited films are weakly crystallized since the major diffracted peaks exhibit low intensity and the average crystallite size calculated from the Scherrer equation is smaller than $15 \mathrm{~nm}$. An increase of the incident angle $\alpha$ up to $40^{\circ}$ leads to more intense peaks for all crystallographic planes, without any preferential orientation. In addition, the crystallite size reaches $30 \mathrm{~nm}$ for $\alpha=40^{\circ}$ and the diffracted patterns (peaks position, intensity or full-width-at-halfmaximum) do not evolve as the incident angle $\alpha$ increases up to $80^{\circ}$. This improved crystallinity as a function of the incident angle has also been observed for other ceramic thin films produced by GLAD [40, 41]. In addition, a reverse effect has been observed by others for some materials [42], showing a reduction of the long range order up to an amorphous structure as the incident angle $\alpha$ rises. As a result, the dependence of crystallinity on the deposition angle has to be considered on a case by case basis and still remains an open question. Nevertheless, it can be correlated with the surface diffusion phenomenon of the sputtered particles. This phenomenon preferentially takes place in the direction of the particle flux, particularly for grazing incident angles. During initial growth and as the incident angle $\alpha$ increases, the formed islands start collecting more adatoms. They will grow faster and tend to capture more incoming vapour flux, reinforcing the growth of large crystallites at the expense of other grains that are consumed during the process. This possible explanation of the long range crystalline order is in agreement with the increase of the crystallite size reported from XRD measurements since grain size rises from 15 to $30 \mathrm{~nm}$ as the incident angle $\alpha$ changes from 0 to $40^{\circ}$, and finally $80^{\circ}$. 


\subsection{Optical characterization}

163 Optical transmittance spectra of tungsten oxide films deposited on glass substrates have been 164 measured in the visible region for various incident angles $\alpha$ of the particle flux (Fig. 3). As expected for $\mathrm{WO}_{3}$ compound, typical interference fringes are observed. The films deposited by conventional process $\left(\alpha=0^{\circ}\right)$ exhibit the highest amplitudes. For a given wavelength (e.g. $\left.600 \mathrm{~nm}\right)$ the envelop curve is below $70 \%$ for the minimum of transmittance $\left(T_{\min }\right)$, whereas it is higher than $91 \%$ for the maximum of transmittance $\left(T_{M a x}\right)$. Amplitude of the fringes is slightly reduced up to an increasing incident angle $\alpha=40^{\circ}$. The amplitudes reduction becomes more significant for grazing incident angles, especially for $\alpha=80^{\circ}$ since $T_{\min }$ is close to $77 \%$ and $T_{\text {Max }}$ is $88 \%$ at $600 \mathrm{~nm}$. For this high incident angle of $80^{\circ}$, it is also worth of noting that fringes tend to disappear as the wavelength comes closer to the absorption edge (i.e. between 400 and $500 \mathrm{~nm}$ ), which can be attributed to the enhancement of the light diffusion. This later is not solely due to structural modification in the film (columns are more inclined), but it also comes from an increased surface roughness for incident angles higher than $40^{\circ}$, as previously observed from SEM analyses (Fig. 1) and in agreement with other theoretical and experimental investigations [43].

From optical transmittance measurements of the $\mathrm{WO}_{3}$ films deposited on glass substrate, refractive index $n$ (Fig. 4) and extinction coefficient $k$ (Fig. 5) have been calculated as a function of the wavelength in the visible region using the Swanepoel's method [44]. The refractive index and extinction coefficient dispersion curves of $\mathrm{WO}_{3}$ films deposited at various incident angles are all fitted by using the Cauchy dispersion equation in the range of wavelengths 400 to $800 \mathrm{~nm}$. Both the optical index and extinction coefficient follow the Cauchy dispersion evolution as a function of wavelength for any incident angle of the particle flux. $\mathrm{WO}_{3}$ thin films prepared with a normal incidence of the particle flux $\left(\alpha=0^{\circ}\right)$ exhibit the highest refractive index together with the lowest extinction coefficient. For a reference wavelength of $589 \mathrm{~nm}, n_{589}=2.17$ (and $k_{589}$ is below $1.42 \times 10^{-}$ ${ }^{3}$ ). This value is below that of the bulk $\mathrm{WO}_{3}$ material since $n_{b u l k}=2.50$ for the same given 
wavelength [45]. It shows that the films sputter deposited at normal incidence are quite compact but nonetheless contain significant amounts of defects and voids.

A systematic change of the incident angle of the particle flux from 0 to $80^{\circ}$ leads to a clear decrease of the refractive index of tungsten oxide thin films from $n_{589}=2.17$ down to 1.90 , respectively. This drop becomes very significant when the incident angle is higher than $40^{\circ}$. This effect has already been observed for other metallic oxide thin films prepared by GLAD [46-48]. It is mainly ascribed to the growth of a more porous structure versus incident angle. In evaporation or sputtering processes, the deposited film's planar density is determined by the shadow length and thus, can be tuned by the incident angle $\alpha$. Varying the amount of bulk material in the film is a way to change its refractive index.

Similarly, extinction coefficient is nearly constant close to $1.50 \times 10^{-3}$ at $589 \mathrm{~nm}$ up to an incident angle of $60^{\circ}$. Hence, it remains close to values corresponding to typical dielectric and transparent compounds. However, for an incident angle of $80^{\circ}$ where $\mathrm{k}_{589}$ is higher than $4.27 \times 10^{-3}$. Such increase of the extinction coefficient correlates with the increase of the surface roughness commonly measured for high incident angles. Indeed, the low values of $k$ in the visible region is a qualitative indication of the good surface smoothness of thin films [49]. Furthermore, the high $k$ value obtained for $\alpha=80^{\circ}$ suggests the presence of marked inhomogeneities in the films (defects, disordering, oxygen vacancies, surface corrugation), especially a rougher film/air interface favoured for high glancing angles of deposition.

The packing density $p$ and, hence, the porosity $\pi$ of the $\mathrm{WO}_{3}$ GLAD films $(\pi=1-p)$ are significant characteristics of sputter deposited materials. They can be calculated based on the effective media approximation, and thus using the mixture rule proposed by Bruggemann [50]:

$\chi_{a}\left(\frac{\varepsilon_{a}-\varepsilon_{e f f}}{\varepsilon_{a}+2 \times \varepsilon_{e f f}}\right)+\chi_{b}\left(\frac{\varepsilon_{b}-\varepsilon_{e f f}}{\varepsilon_{b}+2 \times \varepsilon_{e f f}}\right)=0$

Where $a$ and $b$ components are randomly distributed in space with volume fractions of $\chi_{a}$ and $\chi_{b}$, respectively $\left(\chi_{a}+\chi_{b}=1\right)$. The dielectric properties of the medium are described by an effective 
permittivity $\varepsilon_{\text {eff, }}$, and that of $a$ and $b$ components are $\varepsilon_{a}$ and $\varepsilon_{b}$, respectively. For our films, we considered that $a$ component is the $\mathrm{WO}_{3}$ bulk material and $b$ component is the vacuum. As a result, $\varepsilon_{\text {eff }}$ is the permittivity of the film. Assuming that the bulk tungsten trioxide compound has a refractive index of $n_{b}=2.50$ at $589 \mathrm{~nm}$ [45] and from the refractive index of the film $n_{f}$ at $589 \mathrm{~nm}$, packing density and so, porosity have systematically been calculated and compared to the refractive index as a function of the incident angle $\alpha$ (Fig. 6). Refractive index and porosity exhibit a reverse evolution as the incident angle $\alpha$ rises. $\mathrm{WO}_{3}$ films deposited by conventional incidence $\left(\alpha=0^{\circ}\right)$ show the highest refractive index with $n_{589}=2.18$ and thus, the lowest porosity with $\pi$ lower than $21 \%$. As expected, index is below that of the bulk material because of the total sputtering pressure $(0.18 \mathrm{~Pa})$ used to deposit the films. Thermalisation effect of the sputtered particles and especially, intrinsic low energy bombardment in sputtered thin films are both influenced by the sputtering pressure. They can favour a structure with an open grain boundaries and large columns, leading to a significant void fraction in the deposited film. As a result, density of $\mathrm{WO}_{3}$ deposited film is lower than that of the bulk.

It is also worth of noting that refractive index and porosity are nearly constant up to an incident angle of $50^{\circ}$. Index rapidly drops from $n_{589}=2.14$ down to 1.78 when $\alpha$ changes from 50 to $80^{\circ}$ whereas the porous structure is enhanced and $\pi$ reaches $45 \%$ for $\alpha=80^{\circ}$. It is mainly attributed to the shadowing effect, which prevails on the surface diffusion of adatoms increasing the deposition angle. These results well agree with previous investigations focused on oxide thin films [47, 48]. Varying the amount of bulk material in the film provides a means of tuning its optical properties according to a monotonic and continuous relationship between $n$ and $\alpha$. For highly oblique angles $\left(\alpha>80^{\circ}\right)$, refractive index should approach unity and porosity should tend to $100 \%$. However, the lowest index and thus the maximum porosity for $\mathrm{WO}_{3}$ coatings prepared in this study, obviously depend on the film preparation conditions, but the measurements techniques (spectrometry in transmission by Swanepoel's method, ellipsometry) and environment (humidity) can also influence the reachable index and porosity values. 
238 Because of the peculiar architecture of the GLAD thin films, anisotropic behaviours like 239 birefringence can also be expected. Thus, transmittance spectra were measured with two x and y 240 orthogonal directions of incident linear polarized light $\left(T_{x}\right.$ and $T_{y}$ in the $x$ and $y$ directions, 241 respectively and according to axes defined in Fig. 1). The in-plane birefringence is defined as the 242 difference between the two in-plane refractive indices $\Delta n=n_{x}-n_{y}$, where $n_{x}$ and $n_{y}$ are determined 243 by the Swanepoel's method from $T_{x}$ and $T_{y}$, respectively. Figure 7 illustrates the influence of the incident angle $\alpha$ on the birefringence $\Delta n$ calculated at $617 \mathrm{~nm}$. This birefringence first increases 245 with the incident angle then reaches a maximum value of $\Delta n=0.023$ for $\alpha=50^{\circ}$. The fact that there is an optimised birefringence was also reported by other authors for $\mathrm{ZrO}_{2}$ [42], $\mathrm{ZnS}$ [48], $\mathrm{Ta}_{2} \mathrm{O}_{5}$ [51] or $\mathrm{TiO}_{2}$ [52] films. Furthermore, the value of the maximum $\Delta n$ can be enhanced using a serial 248 bideposition technique as described by Hodginkson and $\mathrm{Wu}$ [51]. For tilted columnar films 249 prepared from standard oblique deposition, the highly porous structure obtained for the highest 250 incident angles does not improve the optical anisotropy. The optimized birefringence can not solely 251 be connected to the porosity, but rather to the biaxial columnar structure. This latter is especially produced for incident angles close to $60^{\circ}$. From simulations and experiments performed by Tait et 253 al. [35], films produce a columnar structure with columns exhibiting an elliptical section versus the 254 incident angle. A structural anisotropy develops parallel to the substrate surface because of the 255 shadowing effect. This effect, mainly in the direction of the incident vapour flux, leads to the 256 formation of growth islands connected to each other by chains perpendicular to the plane of 257 incidence or to the direction of shadowing. The authors established that for an incident angle close 258 to $60^{\circ}$, the shadowing effect prevails on the surface diffusion. By further increasing the $\alpha$ angle, the 259 number of islands falls because of shadowing effect is even more marked. Consequently, the 260 average distance between islands increases. Then, they become disconnected from each other in all 261 directions, resulting in a loss of anisotropy. 
The Swanepoel's method can also be used to calculate the evolution of the absorption coefficient $\xi$ as a function of wavelength. Therefore, the optical band gap $E_{g}$ of $\mathrm{WO}_{3}$ films can be determined from the Tauc's relationship according to the following equation [53]:

$$
\xi h v=C\left(h v-E_{g}\right)^{w}
$$

Where $C$ is a constant and $w$ is $1 / 2,3 / 2,2$ or 3 for transitions being direct and allowed, direct and forbidden, indirect and allowed, and indirect and forbidden, respectively. The values of optical band gap energy $E_{g}$ can be obtained by extrapolating the absorption coefficient to zero absorption in the $(\xi h v)^{1 / w}$ against photon energy $h v$ plot. According to Hjelm et al. [54], $\mathrm{WO}_{3}$ compound exhibits indirect and allowed band gap transitions with $w=2$. Thus, the $E_{g}$ value was extracted from $(\xi h v)^{1 / 2}$ versus $h v$ plot for $\mathrm{WO}_{3}$ films prepared with different incident angles (Fig. 8). Without inclining the particle flux $\left(\alpha=0^{\circ}\right)$, the optical band gap $E_{g}$ is $3.11 \mathrm{eV}$. It is higher than that of the $\mathrm{WO}_{3}$ bulk material, which is $2.62 \mathrm{eV}$ [55] but in agreement with typical values (more than $3 \mathrm{eV}$ ) reported for tungsten trioxide thin films [56]. This high energy gap of oxide thin films compared to the bulk value is mainly associated to the small crystallite size (smaller than $15 \mathrm{~nm}$ from XRD results in Fig. 2). An increase of the incident angle up to $\alpha=60^{\circ}$ does not significantly modify the optical band gap since $E_{g}$ slightly decreases down to $3.05 \mathrm{eV}$. A further increase of the incident angle until $\alpha=$ $80^{\circ}$ leads to reduce $E_{g}$ down to $2.90 \mathrm{eV}$. It can not be ascribed to the improvement of the long range order since it was shown from XRD analyses that the crystallite size reaches $30 \mathrm{~nm}$ for $\alpha=40^{\circ}$ and did not evolve as the incident angle $\alpha$ increased up to $80^{\circ}$ (cf. $\S 3.1$ ). It is rather correlated with an increase of growth and structural defects, which are favoured for high incident angles. Thus, this decrease of the optical band gap for incident angles higher than $60^{\circ}$ could be interpreted as being due to more defects in the film, creating more impurity states in the band gap.

It is also worth of noting that these structural defects and the short range order both facilitate the creation of disorder in the material, favouring a tail of density of states. At lower values of the absorption coefficient $\xi$, the extent of the exponential tail of the absorption edge is characterized by 
287 the Urbach energy $E_{u}$ indicating the width of the band tails of the localized states within the optical 288 band gap. It is given by [57]:

$\xi h v=\xi_{0} \exp \left(\frac{h v}{E_{u}}\right)$

Where $\xi_{0}$ is a constant. It is obvious that the plot of $\ln (\xi)$ versus hv should follow a linear behaviour and allows determining the Urbach energy. This latter was systematically calculated and compared to the optical band gap $E_{g}$ as a function of the incident angle $\alpha$ (Fig. 9). An increase of the Urbach energy from $E_{u}=74$ up to $141 \mathrm{meV}$ corresponds to the decrease in the optical band gap from $E_{g}=$ 3.11 down to $2.90 \mathrm{eV}$ as $\alpha$ rises from 0 to $80^{\circ}$. A linear evolution of $E_{g}$ versus $E_{u}$ can be suggested, which is in agreement with past investigations devoted to thin films [58]. It correlates with an improvement of the crystallinity of the films observed from XRD results and corroborates similar linear evolutions previously obtained by others [59] for films going from the amorphous to the polycrystalline structure. A quantitative relationship between the values of $E_{g}$ and $E_{u}$ under changes in structural site disorder can be determined with linear coefficients closely linked to structural defects in the materials (bond length, bond angle, chemical disorder) [60]. For our $\mathrm{WO}_{3}$ GLAD thin films, the increase of the local disorder as the incident angle rises (increase of $E_{u}$ and reverse evolution of $E_{g}$ ) can be assigned to the secondary grain growth of the voided columnar structure, especially produced for very high incident angles due to a broad incident flux distribution [61]. As a result, the density of defects in the porous structure (e.g. dangling bonds) rises versus the incident angle, leading to higher Urbach energies. 


\section{Conclusion}

Tungsten oxide $\mathrm{WO}_{3}$ thin films with inclined columnar structures were prepared by de reactive magnetron sputtering. The glancing angle deposition technique was implemented to deposit these oriented columnar architectures. Then, a systematic change of the incident angle of the particle flux was performed from $\alpha=0$ to $80^{\circ}$. A clear columnar inclination was produced for incident angles higher than $50^{\circ}$. The resulting columnar angles were tuned from $\beta=0$ to $54^{\circ}$ leading to an emphasized porous microstructure (45\% of porosity) for the most inclined columns. XRD analyses revealed diffracted signals corresponding to the $\mathrm{WO}_{3}$ monoclinic structure with an improved crystallinity as the incident angle increased. Similarly, optical properties like refractive index and extinction coefficient were calculated from optical transmittance measurements in the visible region. Refractive index was significantly reduced from $n_{589}=2.11$ down to 1.90 as the incident angle increased from $\alpha=0$ to $80^{\circ}$. Extinction coefficient remained nearly constant and close to $\mathrm{k}_{589}$ $=1.50 \times 10^{-3}$ up to $\alpha=60^{\circ}$ then became higher than $4.27 \times 10^{-3}$ for the highest incident angles. Variations of the optical behaviours were correlated to the highly porous structure. Voids separating the oriented columns become more significant for incident angles higher than $\alpha=60^{\circ}$ because of the shadowing effect prevailing over the surface adatoms diffusion. Voids and pinholes observed are asymmetric in the $\mathrm{x}$ - and $\mathrm{y}$-directions, which introduce anisotropy and birefringence in thin films. The maximum in-plane birefringence was found to be $\Delta n=0.023$ for an incident angle of $50^{\circ}$. A linear and reverse evolution of the optical band gap versus Urbach energy was noticed with a systematic change of the incident angle, which was correlated with an increase of the density of defects in the highest porous structures.

\section{Acknowledgments}

The authors thank Christine Millot for the SEM observations. The region of Franche-Comté is also acknowledged for the financial support. 


\section{References}

[1] S. Anders, A. Anders, M. Rubin, Z. Wang, S. Raoux, F. Kong, I.G. Brown, Surf. Coat. Technol., 76(1-3) (1995) 167-173.

[2] C.G. Granqvist, Solar Energy Materials and Solar Cells, 91(17) (2007) 1529-1598.

[3] G.A. Niklasson, C.G. Granqvist, J. Mater. Chem., 17(2) (2007) 127-156.

[4] J.N. Yao, Y.A. Yang, B.H. Loo, J. Phys. Chem. B, 102(11) (1998) 1856-1860.

[5] K. Aguir, C. Lemire, D.B.B. Lollman, Sensor Actuat. B, 84(1) (2002) 1-5.

[6] G. Sberveglieri, L. Depero, S. Groppelli, P. Nelli, Sensor Actuat. B, 26(1-3) (1995) 89-92.

[7] G. Eranna, B.C. Joshi, D.P. Runthala, R.P. Gupta, Crit. Rev. Solid State, 29(3-4) (2004) 111 188.

[8] D. Manno, A. Serra, M. Di Giulio, G. Micocci, A. Tepore, Thin Solid Films, 324(1-2) (1998) 44-51.

[9] S.H. Mohamed, H.A. Mohamed, H.A. Abd El Ghani, Physica B, 406(4) (2011) 831-835.

[10] W. Wagner, F. Rauch, R. Feile, C. Ottermann, K. Bange, Thin Solid Films, 235(1-2) (1993) 228-233.

[11] B.G. Lewis, D.C. Paine, MRS Bulletin August, 25 (2000) 22-27.

[12] M. Seman, C.A. Wolden, J. Vac. Sci. Technol., A21 (2003) 1927-1933.

[13] N. Martin, A.R. Bally, P. Hones, R. Sanjinès, F. Lévy, Thin Solid Films, 377 (2000) 550-556.

[14] P. Carvalho, L. Cunha, E. Alves, N. Martin, E. Le Bourhis, F. Vaz, J. Phys. D: Appl. Phys., 42(19) (2009) 195501-7.

[15] S.H. Lee, H.M. Cheong, C.E. Tracy, A. Mascarenhas, A.W. Czanderna, S.K. Deb, Appl. Phys. Lett., 75(11) (1999) 1541-1543.

[16] A.R. Bally, P. Hones, R. Sanjinès, P.E. Schmid, F. Lévy, Surf. Coat. Technol., 108(1-3) (1998) 166-170.

[17] M.J. Brett, M.M. Hawkeye, Science, 319 (2008) 1192-1193. 
[18] G. Cao, Y. Wang, Nanostructures and nanomaterials - Synthesis, Properties and Applications, World Scientific Publishing Co. Pte. Ltd., Singapore (2011).

[19] K. Robbie, M.J. Brett, A. Lakhtakia, Nature, 384 (1999) 616.

[20] K. Robbie, L.J. Friedrich, S.K. Dew, T. Smy, M.J. Brett, J. Vac. Sci. Technol., A13(3) (1995) 1032-1035.

[21] Y. He, J. Fu, Y. Zhang, Y. Zhao, L. Zhang, A. Xia, J. Cai, Small, 3(1) (2007) 153-160.

[22] K. Robbie, J.C. Sit, M.J. Brett, J. Vac. Sci. Technol., B16(3) (1998) 1115-1122.

[23] K. Robbie, D.J. Broer, M.J. Brett, Nature, 399 (1999) 764-766.

[24] A. Van Popta, J.C. Sit, M.J. Brett, Proc. of SPIE, 5464 (2004) 198-208.

[25] P.M. Martin, B.F. Monzyk, E.C. Burckle, J.R. Busch, R.J. Gilbert, K.A. Dasse. 2005, Mater. Sci. Eng., B119 (2005) 246-251.

[26] M.M. Hawkeye, R. Joseph, J.C. Sit, M.J. Brett, Opt. Express, 18 (2010) 13220-13226.

[27] V. Kesapragada, P. Victor, O. Nalamasu, D. Gall, Nano Lett., 6 (2006) 854-857.

[28] K. Kaminska, T. Brown, G. Beydaghyan, K. Robbie, Appl. Optics, 42 (2003) 4212-4219.

[29] Y.J. Park, D.H. Chang, C.K. Hwangbo, Pacific Rim Conference on Lasers and Electro-optics, $1-4(2007) 33-34$.

[30] S.R. Kennedy, M.J. Brett, Appl. Optics, 42 (2003) 4573-4579.

[31] J. Guillot, J.M. Chappé, O. Heintz, N. Martin, L. Imhoff, J. Takadoum, Acta Mater., 54(11) (2006) 3067-3074.

[32] N. Martin, J. Lintymer, J. Gavoille, J.M. Chappé, F. Sthal, J. Takadoum, F. Vaz, L. Rebouta, Surf. Coat. Technol., 201(18) (2007) 7720-7726.

[33] G. Beydaghyan, G. Bader, P.V. Ashrit, Thin Solid Films, 516(8) (2008) 1646-1650.

[34] P.C.P. Hrudey, M. Taschuk, Y.Y. Tsui, R. Fedosejevs, J.C. Sit, M.J. Brett, Proceedings of International Conference on MEMS, NANO and Smart Systems, (2003) 327-331.

[35] R.N. Tait, T. Smy, M.J. Brett, J. Vac. Sci. Technol., A10(4) (1992) 1518-1521.

[36] J.M. Nieuwenhuizen, H.B. Haanstra, Philips Techn. Rev., 27(3-4) (1966) 87-91. 
383 [37] J. Lintymer, N. Martin J.M. Chappé, J. Takadoum, P. Delobelle, Thin Solid Films, 503(1) 384 (2006) 177-189.

385 [38] A. Besnard, N. Martin, L. Carpentier, B. Gallas, J. Phys. D: Appl. Phys., 44 (2011) 215301-8. 386 [39] R.N. Tait, T. Smy, M.J. Brett, Thin Solid Films, 226(2) (1993) 196-201.

387 [40] N.J. Gerein, M.D. Fleischauer, M.J. Brett, Solar Energy Materials and Solar Cells, 94(12) 388 (2010) 2343-2350.

389 [41] A.R. Shetty, A. Karimi, M. Cantoni, Thin Solid Films, 519 (2011) 4262-4270.

390 [42] S. Wang, X. Fu, G. Xia, J. Wang, J. Shao, Z. Fan, Appl. Surf. Sci., 252 (2006) 8734-8737.

391 [43] A. Dolatshahi-Pirouz, M.B. Hovgaard, K. Rechendorff, J. Chevallier, M. Foss, F. Besenbacher, 392 Phys. Rev. B, 77 (2008) 115427-5.

393 [44] R. Swanepoel, J. Phys. E: Sci. Instrum., 16 (1983) 1214-1222.

394 [45] R. Sawada, G.C. Danielson, Phys. Rev., 113 (1959) 1008-1013.

395 [46] S. Wang, X. Zhao, Z. Fan, J. Shao, Appl. Phys. A, 107 (2012) 227-232.

396 [47] K.M. Sobahan, Y.J. Park, C.K. Hwangbo, J. Korean Phys. Soc., 55 (2009) 1272-1277.

397 [48] S. Wang, G. Xia, X. Fu, H. He, J. Shao, Z. Fan, Thin Solid Films, 515 (2007) 3352-3355.

398 [49] S. Bhaskar, S.B. Majumder, M. Jain, P.S. Dobal, R.S. Katiyar, Mater. Sci. Eng. B, 87 (2001) $399 \quad 178-190$.

400 [50] S. Kasap, P. Capper, Springer Hanbook of Electronic and photonic materials, New York; 401 Berlin: Springer, 2006.

402 [51] I.J. Hodgkinson, Q.H. Wu, Appl. Optics, 38 (1999) 3621-3625.

403 [52] S.H. Woo, K. Hwangbo, J. Korean Phys. Soc., 49 (2006) 2136-2142.

404 [53] J. Tauc, R. Grigorovici, A. Vancu, Physics State Solid, 15 (1966) 627-637.

405 [54] A. Hjelm, C.G. Granqvist, J.M. Wills, Phys. Rev. B, 54 (1996) 2436-2445.

406 [55] F.P. Koffyberg, K. Dwight, A. Wold, Solid State Commun., 30 (1979) 433-437.

407 [56] S.H. Mohamed, H.A. Mohamed, H.A. Abd El Ghani, Physica B, 406 (2011) 831-835.

408 [57] S. Zaynobidinov, R.G. Ikramov, R.M. Jalalov, J. Appl. Spectrosc., 72 (2011) 211-227. 
409 [58] M. Caglar, S. Ilican, Y. Caglar, Y. Sahin, F. Yakuphanoglu, D. Hur, Spectrochim. Acta A, 71 $410 \quad$ (2008) 621-627.

411 [59] J. Melsheimer, D. Ziegler, Thin Solid Films, 129 (1985) 35-47.

412 [60] A.S. Ferlauto, G.M. Ferreira, J.M. Pearce, C.R. Wronski, R.W. Collins, X. Deng, G. Ganguly, $413 \quad$ J. Appl. Phys., 92 (2002) 2424-2436.

414 [61] Y.G. Yang, D.D. Hass, H.N.G. Wadley, Thin Solid Films, 71 (2005) 1-11. 


\section{Figure captions}

$416 \quad$ Figure 1

417 Surface and cross-section observations by $\mathrm{SEM}$ of $\mathrm{WO}_{3}$ thin films sputter deposited on (100) Si 418 with two different incident angles $\alpha$ of the sputtered particles: a) and b) $\alpha=70^{\circ}$; c) and d) $\alpha=80^{\circ}$. 419 Direction of incoming particle flux, incident angle $\alpha$, column angle $\beta$ and $(x, y, z)$ axes are 420 indicated. The scale bar is the same for all images.

422 Figure 2

423 X-ray diffraction patterns of the tungsten oxide thin films deposited on (100) Si with various 424 incident angles $\alpha$ of the particle flux $\left(\alpha=0,40\right.$ and $\left.80^{\circ}\right)$. Diffracted signals $\left(^{*}\right)$ corresponding to the 425 monoclinic $\mathrm{WO}_{3}$ structure are detected $(\mathrm{Si}=$ silicon substrate $)$.

427 Figure 3

428 Optical transmittance spectra in the visible range of tungsten oxide thin films deposited on glass 429 substrate for incident angles $\alpha=0,40$ and $80^{\circ}$. Clear interference fringes are measured, which are 430 typical of transparent thin films.

$432 \quad$ Figure 4

433 Refractive index $\mathrm{n}$ as a function of wavelength $\lambda$ in the visible range for tungsten oxide thin films 434 deposited on glass substrate with incident angles $\alpha=0,40,60$ and $80^{\circ}$. A Cauchy dispersion law 435 was used to fit the evolution of $n$ versus $\lambda$.

Figure 5

438 Extinction coefficient $k$ as a function of wavelength $\lambda$ in the visible range for tungsten oxide thin 439 films deposited on glass substrate with incident angles $\alpha=0,40,60$ and $80^{\circ}$. 
Figure 6

442 Refractive index $n_{589}$ at $\lambda=589 \mathrm{~nm}$ and porosity $\pi$ of $\mathrm{WO}_{3}$ thin films versus incident angle $\alpha$. 443 Porosity was determined from the packing density based on the Bruggemann effective medium 444 approximation. Incident angles higher than $50^{\circ}$ lead to the most significant changes of the refractive 445 index and porosity. Dashed lines are guides for the eye.

$447 \quad$ Figure 7

448 In-plane birefringence $\Delta n$ at $\lambda=617 \mathrm{~nm}$ for $\mathrm{WO}_{3}$ thin films as a function of the incident angle $\alpha$. A 449 maximum of anisotropy is obtained for an incident angle of $50^{\circ}$. Dashed line is guide for the eye.

450

$451 \quad$ Figure 8

452 Typical plot of the absorption coefficient $(\alpha h v)^{1 / 2}$ versus photon energy $h v$ for $\mathrm{WO}_{3}$ thin films 453 prepared for incident angles $\alpha=0,40$ and $80^{\circ}$. Indirect and allowed transitions were assumed to 454 deduce the optical band gap according to the Tauc's relationship. Solid lines in the figure refer to 455 extrapolation for determining the optical band gap.

$457 \quad$ Figure 9

458 Linear evolution of the optical band gap $E_{g}$ as a function of Urbach energy $E_{u}$ of $\mathrm{WO}_{3}$ thin films 459 deposited with a systematic increase of the incident angle $\alpha$ from 0 to $80^{\circ}$. Dashed line is guide for 460 the eye. 
Figure 1
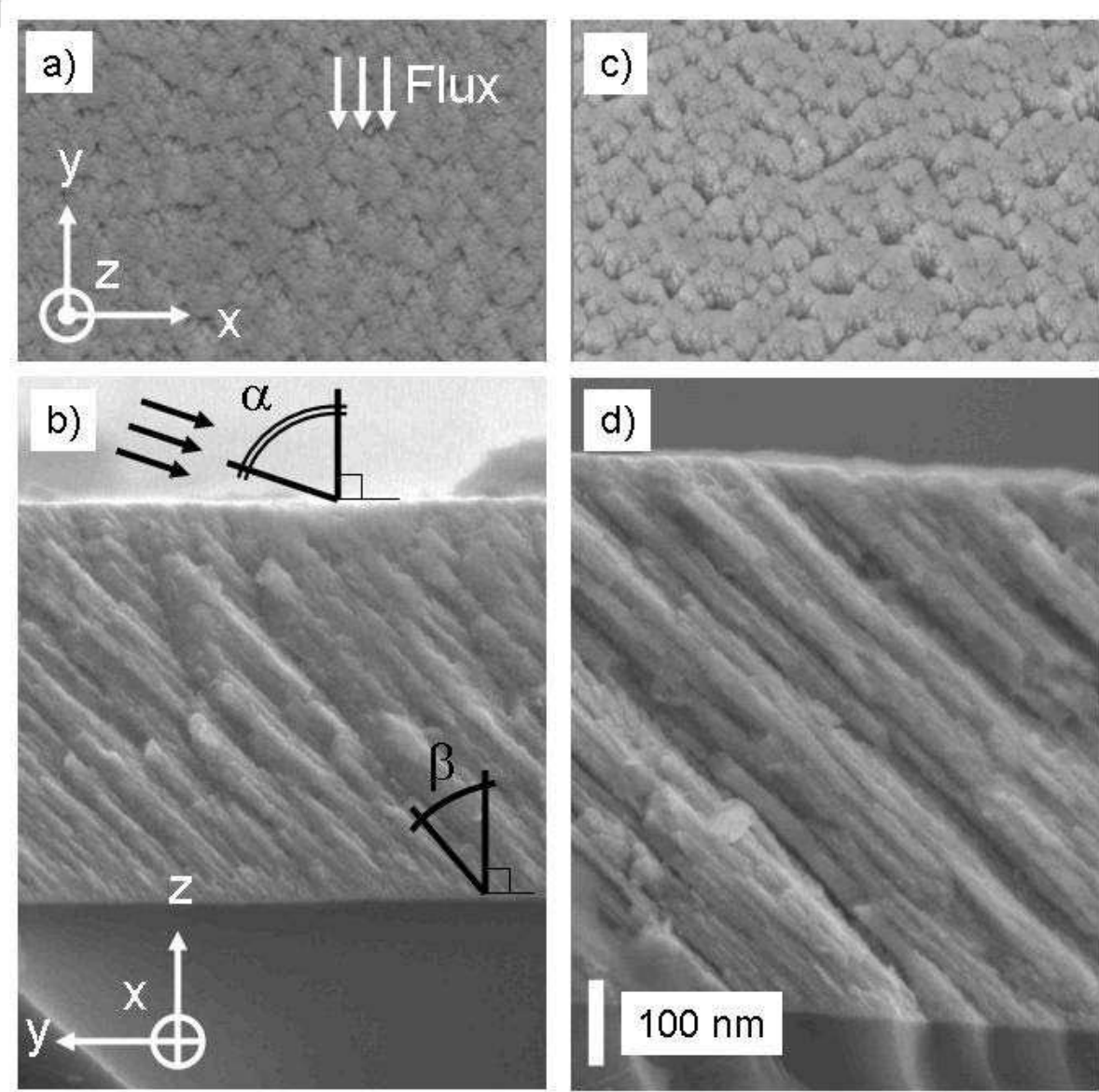
Figure 2

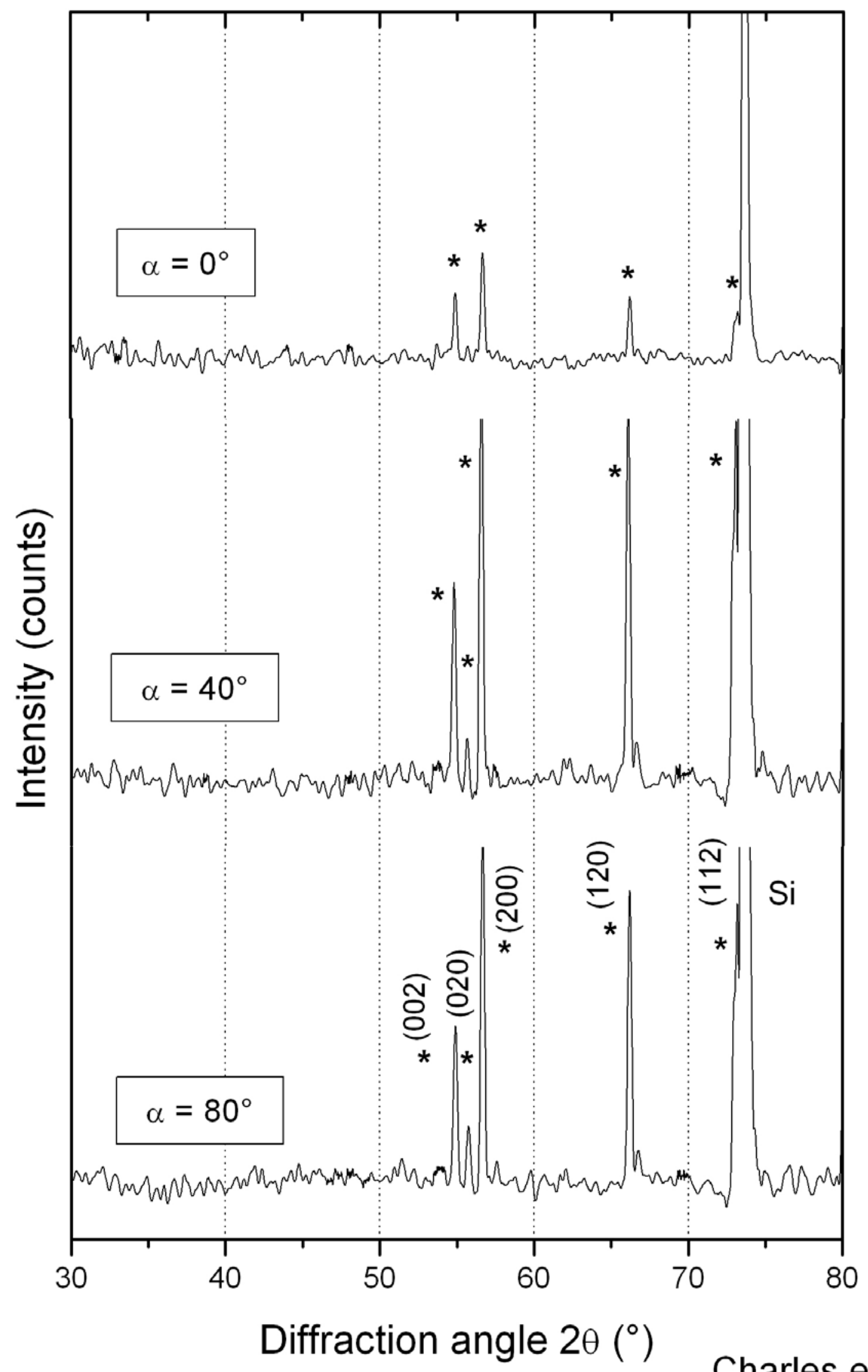


Figure 3

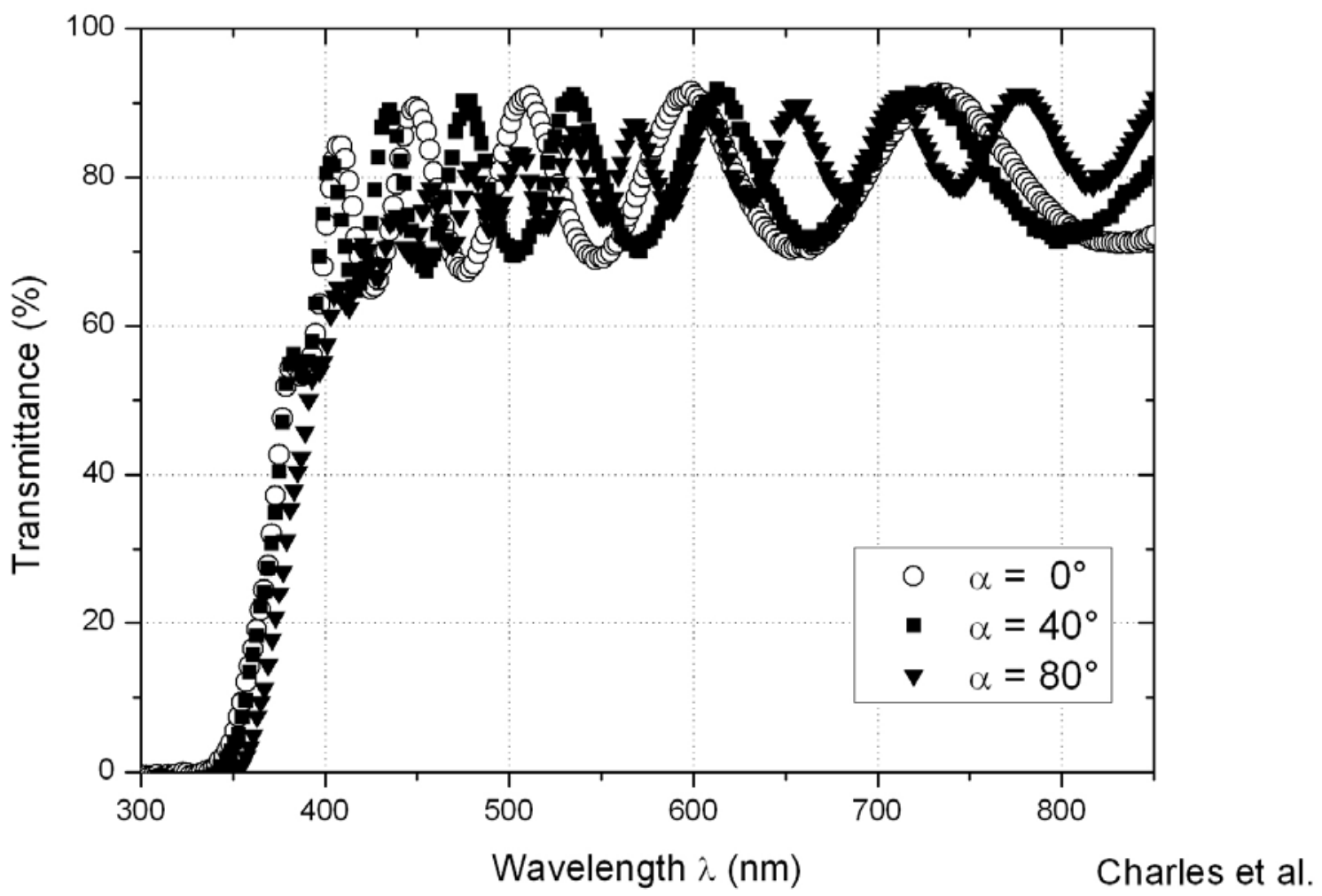


Figure 4

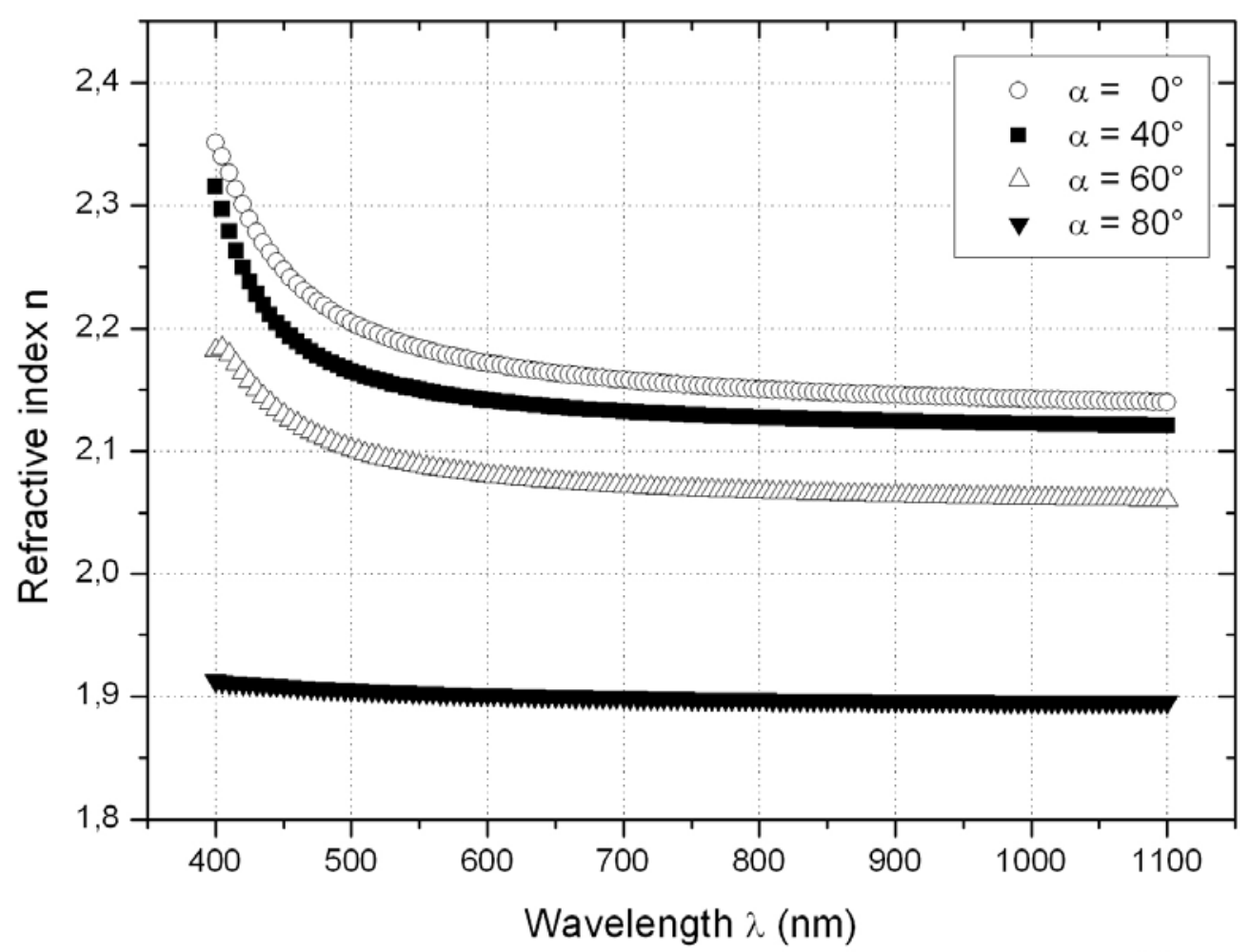


Figure 5

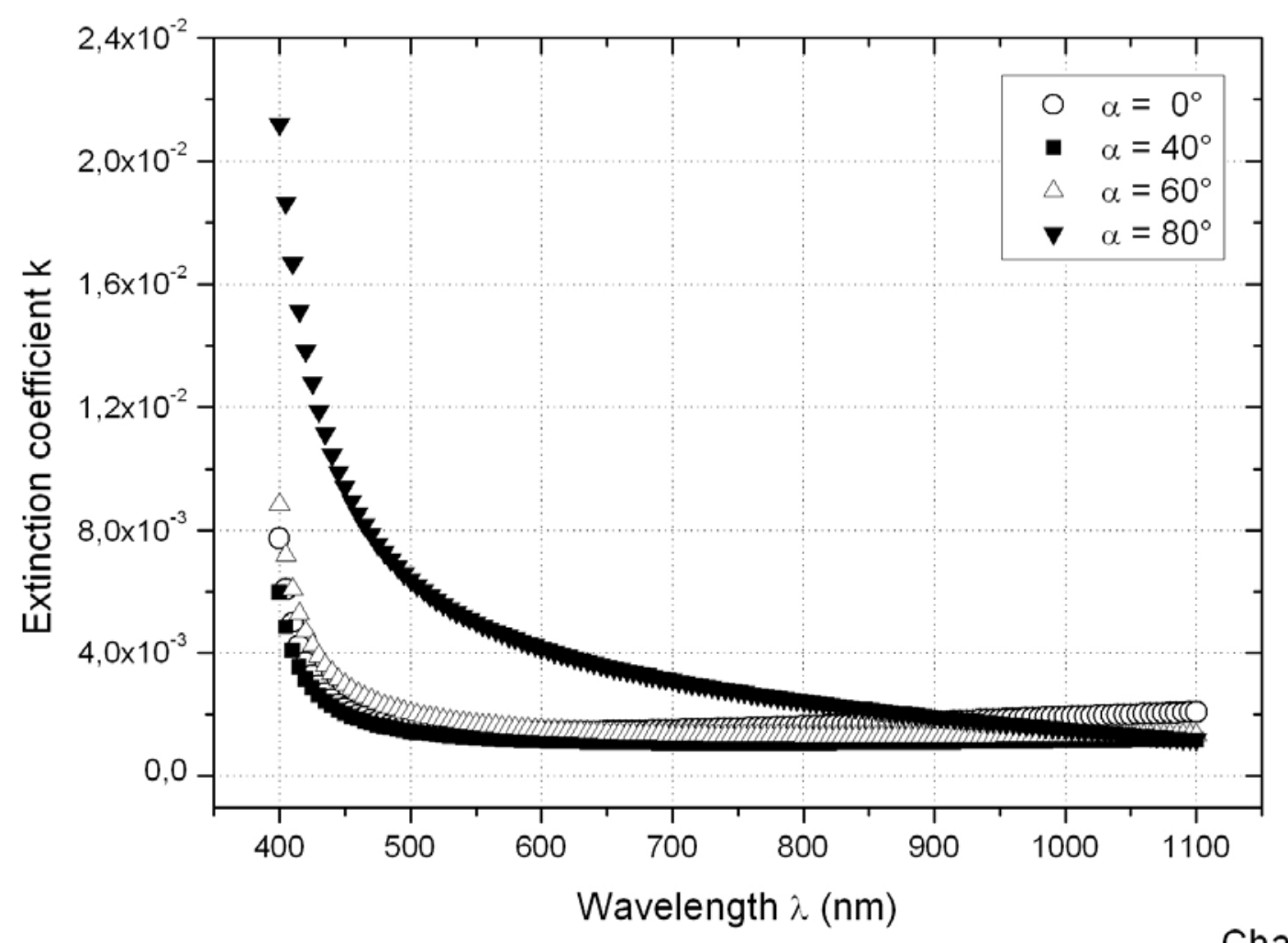

465

Charles et al. 
Figure 6

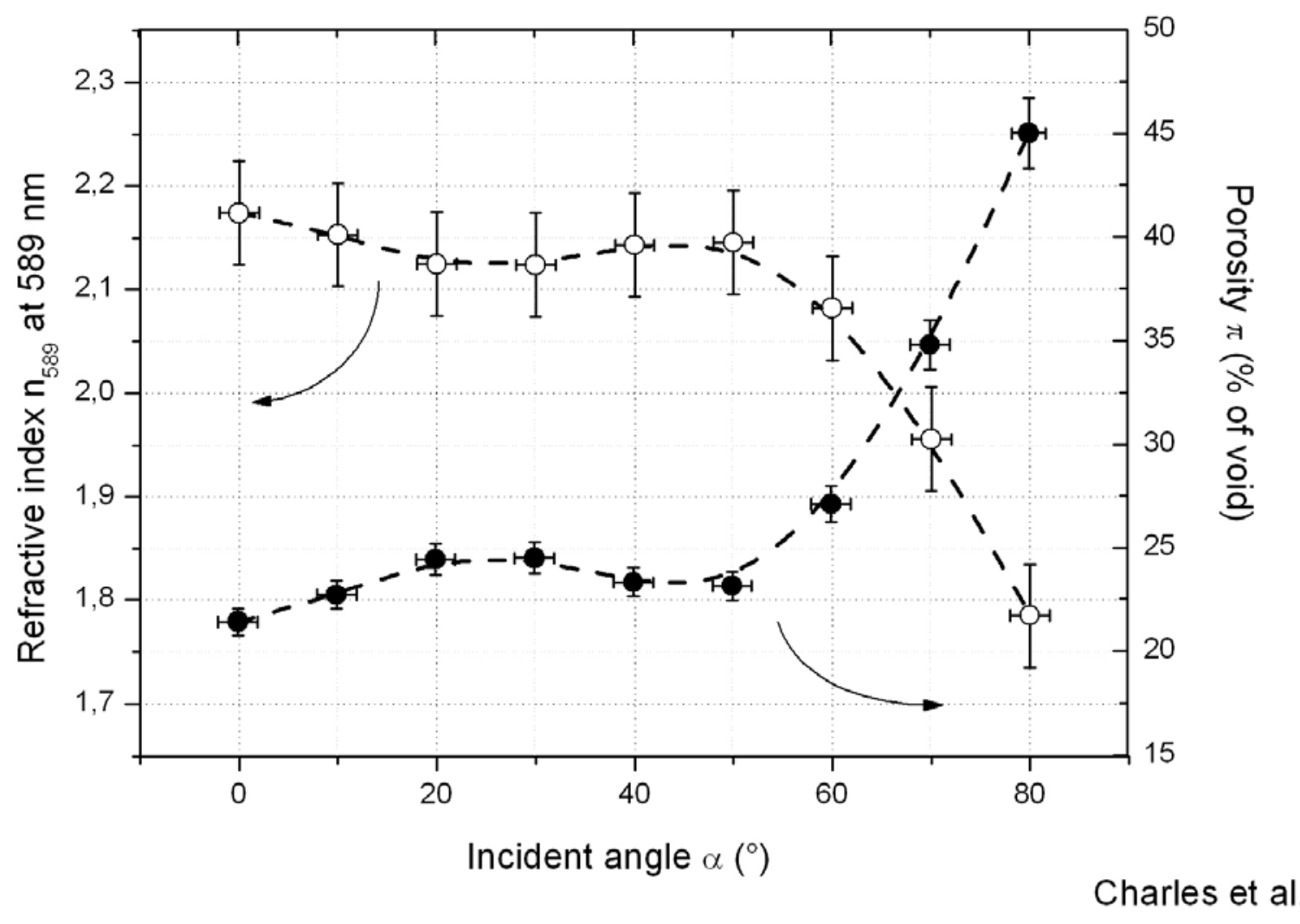


Figure 7

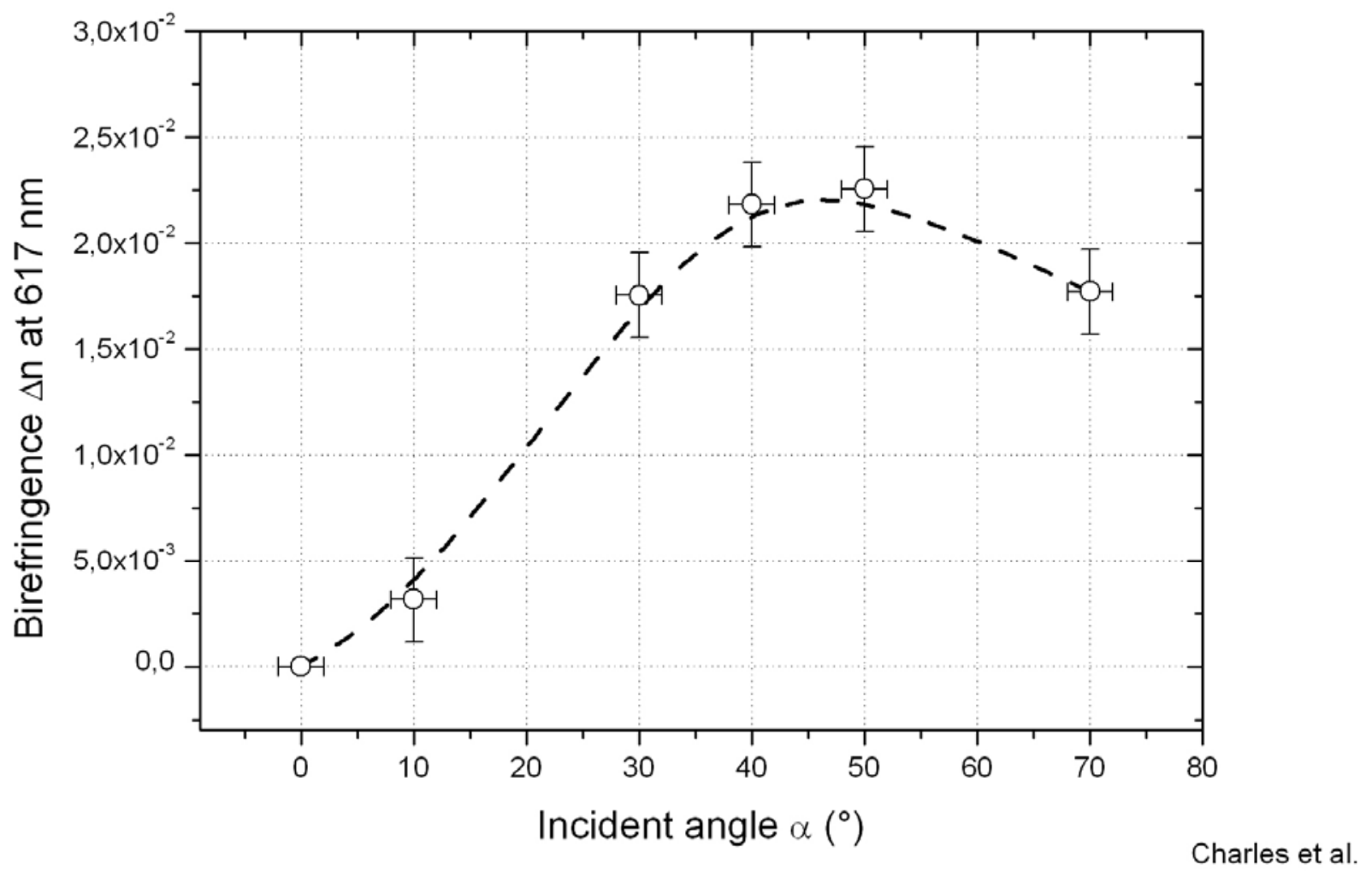


Figure 8

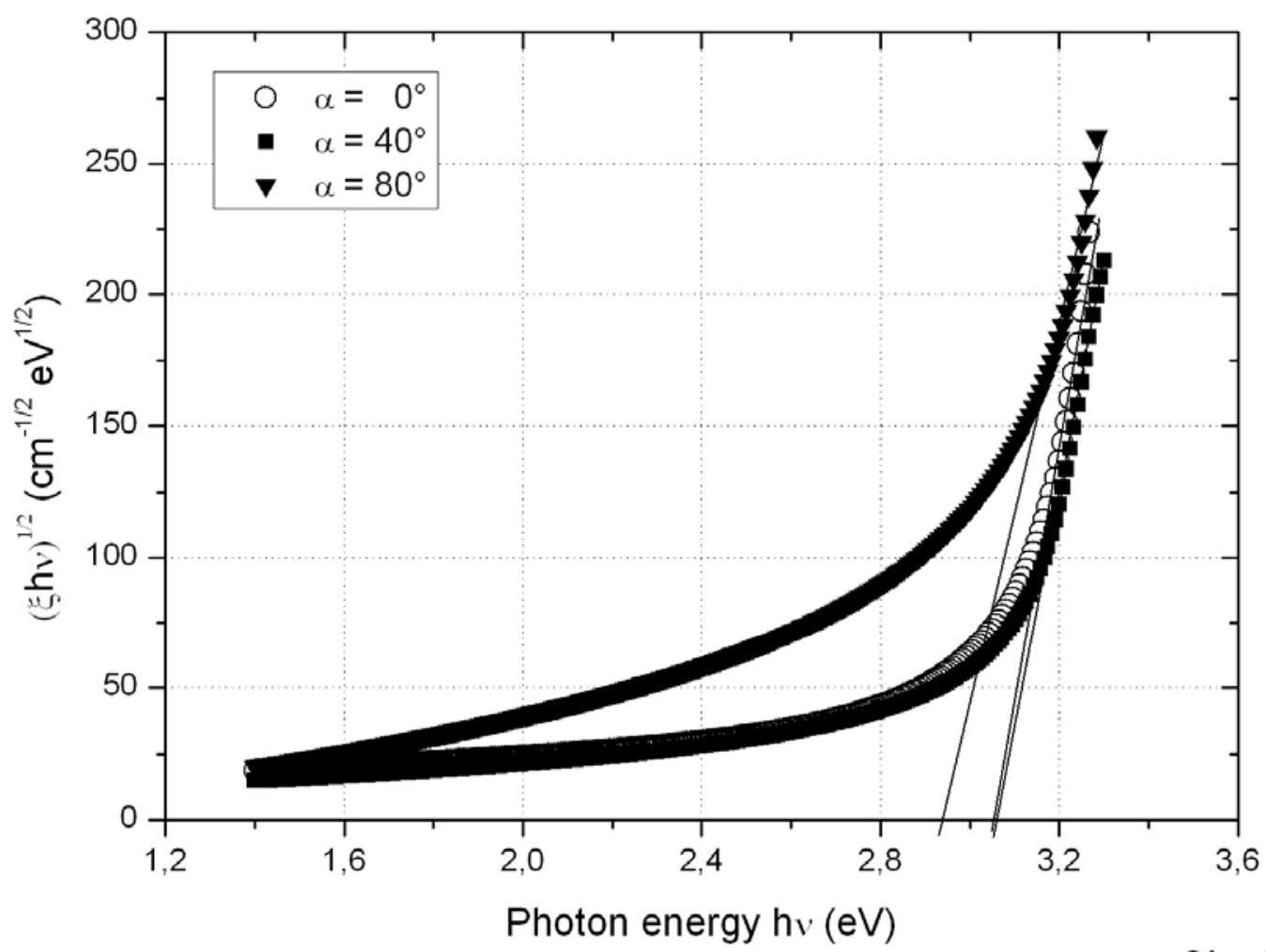

Charles et al. 
Figure 9

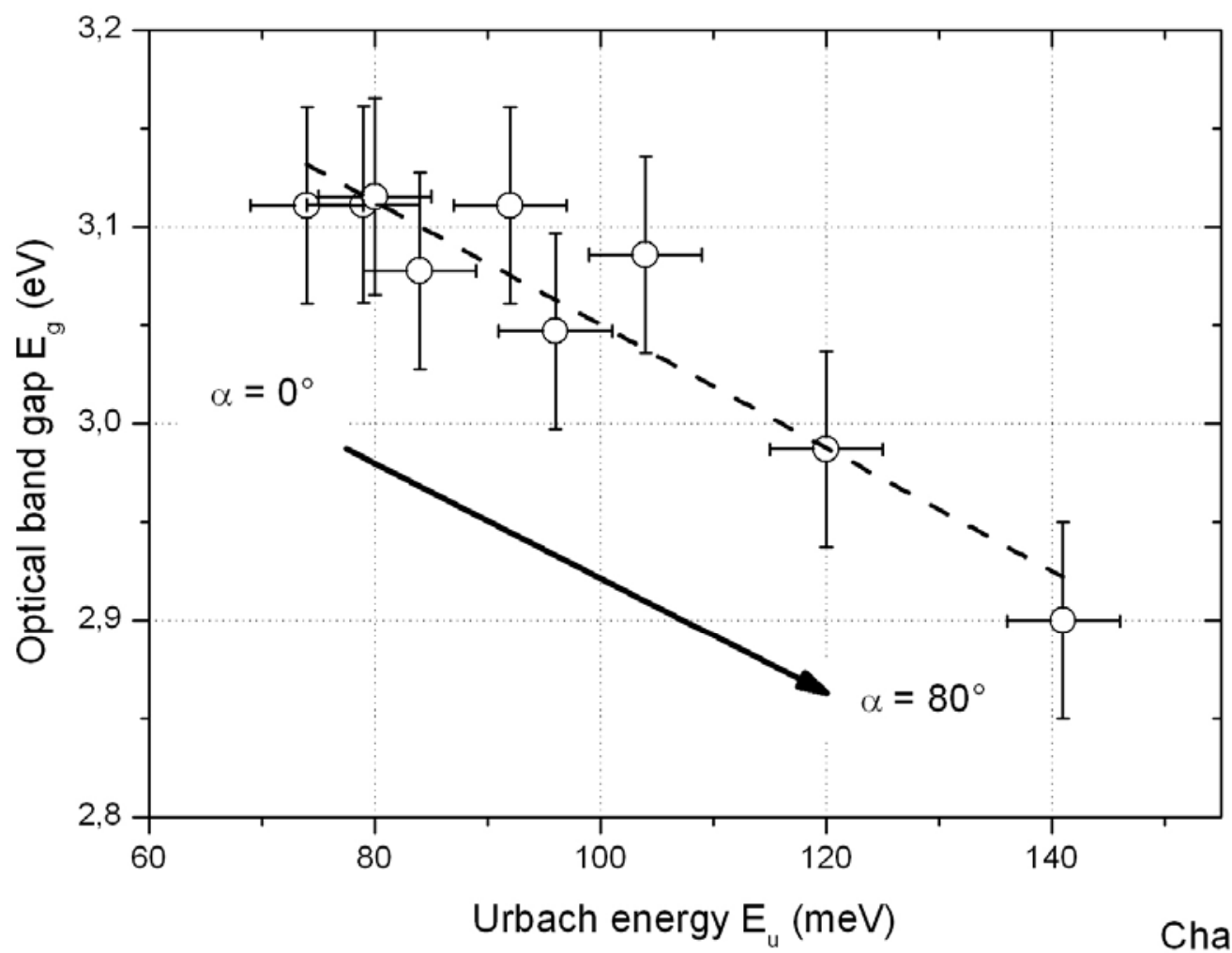

